\title{
INCOHERENT INFRARED SPATIAL INTERFEROMETRY
}

Frank J Low

Steward Observatory and Lunar and Planetary Laboratory, University of Arizona, Tucson, Arizona 85721 USA

\section{ABSTRACT}

By adapting the principles of interferometry first developed by Michelson, we have built a simple incoherent interferometer which operates efficiently from 2 to 20 microns

The design and performance of this instrument will be discussed More than 12 stars have been at least partially resolved at one or more wavelengths and, in several cases, their sizes and shapes have been determined A number of objects in the solar system have also been studied and useful studies of extragalactic nuclei are now possible. With the development of the new Multiple Mirror Telescope both the resolution and the sensitivity of this instrument will soon be extended. 


\section{INTRODUCTION}

With the immediate objective of increasing the angular resolution of

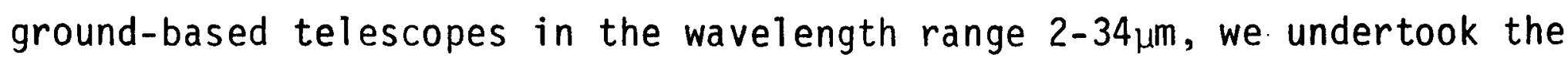
development of an incoherent spatial interferometer based on Michelson's original concept. Our instrument was first operated successfully at $5 \mu \mathrm{m}$ in Jan $1975^{1}$ It can now be used routinely with various telescopes at $2.2,3.4,5.0,8,10,11,12.5$, and $22 \mu \mathrm{m}$ to achieve angular resolution in the range 5-0.05 arcsec on objects at least as faint as zero magnitude (40 Jy at $10 \mu \mathrm{m})$.

The design, operation and performance of the instrument in its present configuration will be discussed and a few astronomical results will be described Additional results will be described by $R$. Howell and by D. McCarthy later in the program. It will be seen that further improvement is needed before the efficiency and sensitivity of the instrument reaches fundamental limits our current efforts in this area will be outlined In conclusion, the exciting project of adapting this technique to the new 6.9 meter Multiple Mirror Telescope (MMT) nearing completion on Mt Hopkins, south of Tucson, will indicate one of several ways in which the angular resolution at infrared wavelengths can be extended even farther

\section{DESIGN OF THE INTERFEROMETER}

Figure 1 shows the optical configuration of our instrument as it is used at the Cassegrain Focus of various telescopes More details are given by McCarthy et al ${ }^{2}$ Note that fringe modulation is achieved by varying the relative pathlength using the two piezoelectrically driven mirrors Both square wave modulation and continuous scanning are possible and have been used. Normally the mirror is driven by a $40 \mathrm{~Hz}$ sawtooth waveform which produces a linear scan of the fringe pattern over the grid placed at the focus. The amplitude of the modulation is adjusted for each wavelength so that a sinusoidal fringe signal at $40 \mathrm{~Hz}$ is produced. When the wavelength is changed the grid must also be changed to match the fringe pattern. 


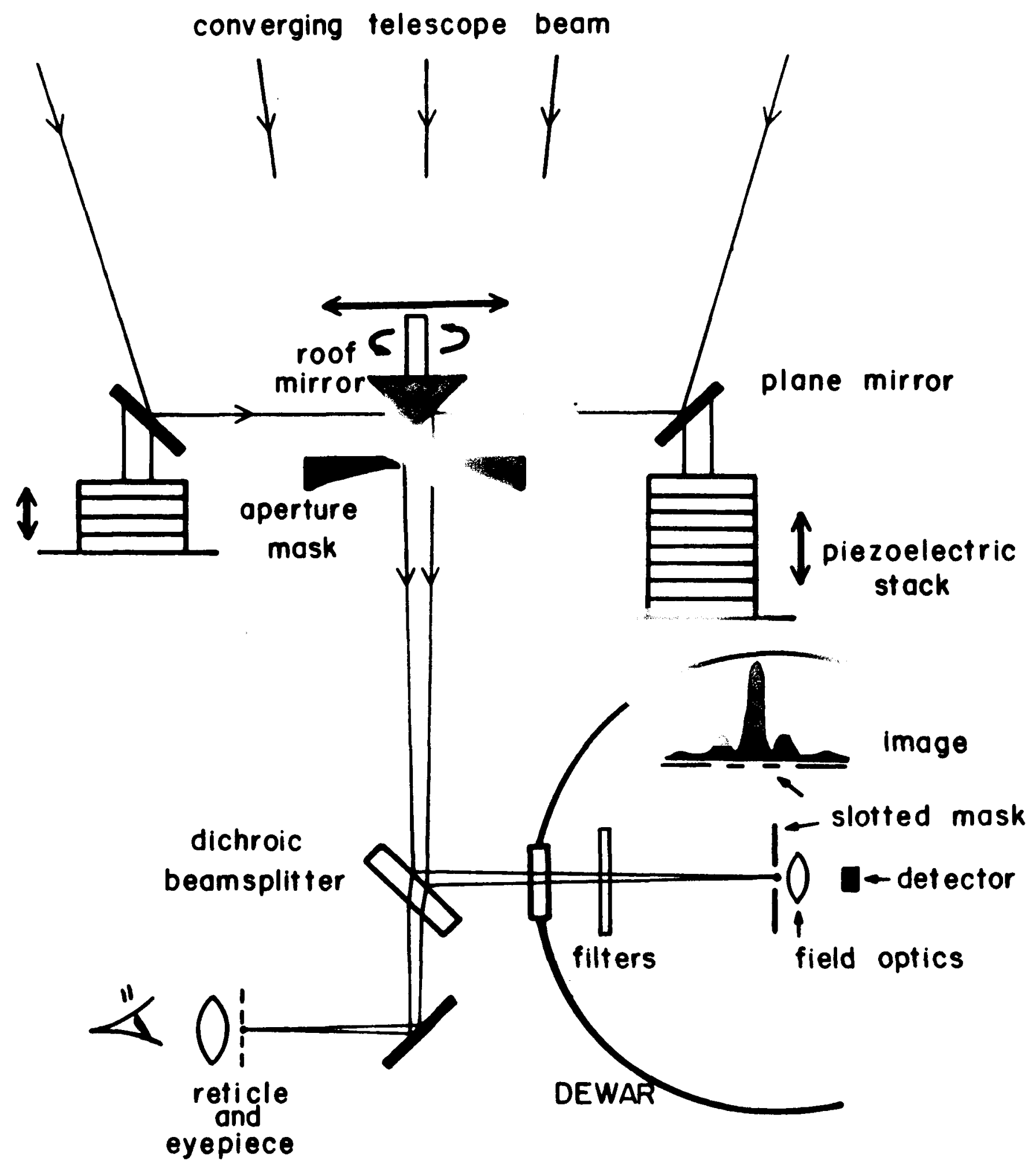

Figure 1 Optical configuration of the interferometer developed by McCarthy and Low at the University of Arizona 
The beams may also be spatially modulated at $20 \mathrm{~Hz}$ by rotating the roof-mirror through an angle of about 0.5 degrees Then the total power in each beam can be measured and the instrument functions as an infrared photometer

The dewar contains the detector (a Ge bolometer operated at $1.2 \mathrm{~K}$ for 5-22 $\mu \mathrm{m}$ or an InSb photovoltaic detector operated at $77 \mathrm{~K}$ for $2-5 \mu \mathrm{m})$, a field mirror operated as a Fabry lense, the grid as shown, and the cooled interference filter to select the desired band of wavelengths

In practice it is not feasible to change the aperture spacing while observing, so we use different telescopes to vary the spacing as follows-$0.9,1.2,19$, and 3.2 meters It is quite feasible, however, to rotate the instrument at the Cassegrain focus to obtain all position angles on the sky

\section{OPERATION OF THE INSTRUMENT}

Alignment and path-length equalization is carried out visually using a bright star to observe fringes With the fringe modulator in operation a "sinusoidal" signal is produced as in Figure 2 The effects of "seeing" are apparent as randomly varying changes in frequency and phase. This signal is filtered by a $40 \mathrm{~Hz}$ analogue bandpass filter which must be wide enough to pass all the frequencies generated by "seeing" R. Howell will discuss the quantitative effects of "seeing" in his paper

There are two signal processing paths, one analogue and one digital In the analogue system a phase-locked-loop tracks the fringe signal and drives a phase-sensitive detector and strip chart recorder with a $1 \mathrm{sec}$ time constant. This is used for real-time monitoring and system check out. In the digital system the digitized signal is recorded along with the scan signal for future processing but it is also analyzed by a small computer which calculates the rms power in the fringe signal for a specified period of $10 \mathrm{sec}$. The noise power is subtracted by moving the telescope to empty sky for an equal period after each $10 \mathrm{sec}$ integration. 


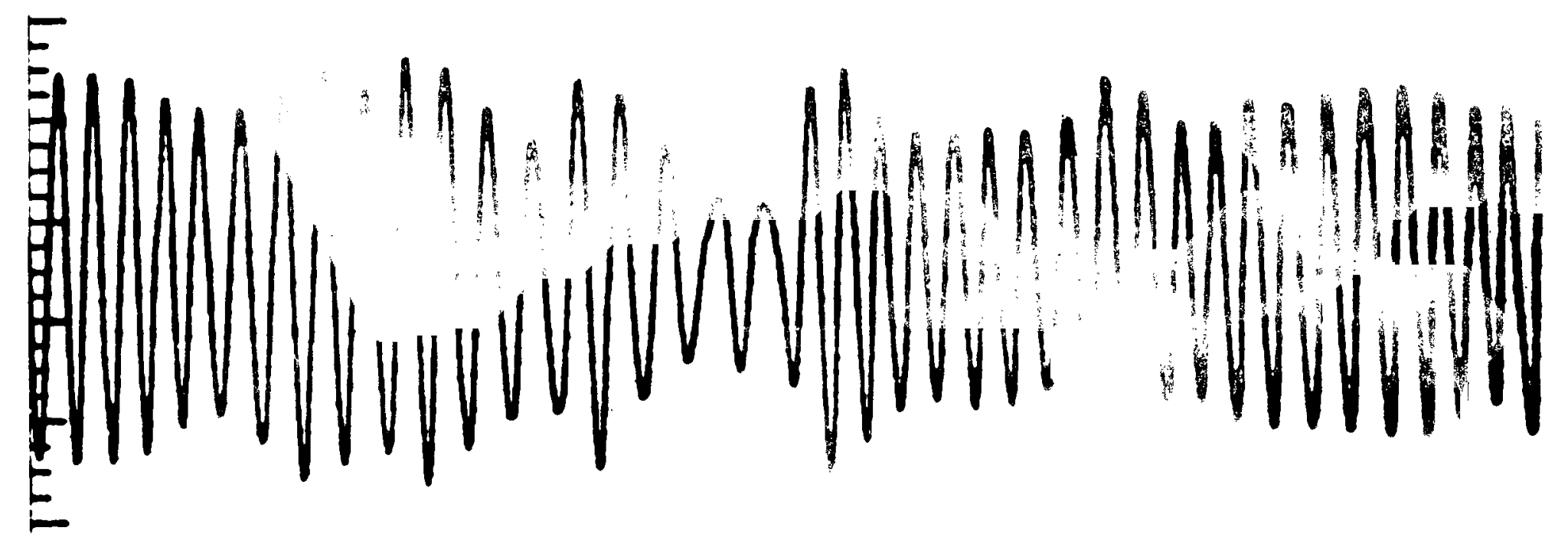

Figure 2. Recording of the $\sim 40 \mathrm{~Hz}$ fringe signal showing effects of "seeing"

Instrumental effects are calibrated by observing point sources several times during the night First the star is observed photometrically, then the fringe signal is measured The ratio of these signals, which are measured sequentially on a time scale of minutes, may change during the night as the "seeing" changes or if the instrumental alignment changes. Thus frequent measurements of "point sources" or "known sources" must be made if reliable results are to be obtained.

\section{PERFORMANCE}

In principle our $S / N$ for a bright object in good "seeing" should approach that of a background limited photometer of equal collecting area after correcting for losses in the two additional mirrors and in the grid In practice we are not yet background limited at $10 \mu \mathrm{m}$ but are somewhat closer at $5 \mu \mathrm{m}$. Table 1 gives the instrumental parameters, theoretical performance and measured performance for a night of good "seeing" ( $\sim 1$ arcsec visual) at $10.2 \mu \mathrm{m}$ on the Kitt Peak National Observatory 3.8 meter telescope

In section 6 our current efforts to improve the performance will be discussed. Here it may be noted that with an integration time of 15 
Table 1 Instrumental Parameters and Performance

\begin{tabular}{|c|c|}
\hline Baseline & $3.2 \mathrm{~m}$ \\
\hline Aperture & $0.64 \mathrm{~m}$ \\
\hline$\lambda_{C}$ & $10.2 \mu \mathrm{m}$ \\
\hline$(S . N)_{T h}^{*}$ & 570 at $N=-2.5$ \\
\hline$(S / N)_{\text {Obs }}^{*}+\dagger$ & 10 at $\mathrm{N}=-2.5 \Rightarrow 400 \mathrm{Jy} \Rightarrow 1 \times 10^{-15} \mathrm{w} / \mathrm{cm}^{2} / \mu \mathrm{m}$ \\
\hline $\begin{array}{l}\text { Fringe Mod } \\
\text { Eff }\end{array}$ & 0.98 \\
\hline \multicolumn{2}{|c|}{$\begin{array}{l}\text { Peak signal/rms noise measured with phase sensitive detector } \\
\text { with } 1 \text { sec integration time. }\end{array}$} \\
\hline \multicolumn{2}{|c|}{$\begin{array}{l}+ \text { Assumptions are, grid eff }=.5 \text {, optical eff }=.8 \text {, instrumen } \\
\text { plus sky emissivity at } 260 \mathrm{~K}=0 . \text { This implies signal of } \\
2 \times 10^{-12} \mathrm{w} \text { and background } 1 \text { imited NEP }=3.5 \times 10^{-15} \mathrm{~W} / \mathrm{Hz}^{\frac{1}{2}} \text {. } \\
+ \text { Measured NEP }=3 \times 10^{-14} \mathrm{~W} / \mathrm{Hz}^{\frac{1}{2}} \text {. }\end{array}$} \\
\hline
\end{tabular}

minutes, equally divided between star and sky, a measurement of Vega at $10 \mu \mathrm{m}$ gave a fringe amplitude of $40 \pm 8$ Jy in moderate "seeing" of 2 arcsec Since the phase of a weak signal cannot be measured in real time, the time required to produce a given $S / N$ ratio decreases as the fourth power of source intensity As "seeing" forces a wider bandwidth of the analogue filter to be used, the $S / N$ degrades as $\Delta f^{\frac{1}{2}}$

In the above discussion it is assumed that the "seeing" effects are small enough that coherence is not lost over the diameter of the two apertures In our case the largest aperture diameter used is $0.74 \mathrm{~m}$ and no loss of coherence is found at 10um when the "seeing" is 3 arcsec or better When the "seeing" is worse than 3 arcsec the rate of change and/or amplitude of change of phase in the fringe signal become too large and the accuracy 
and repeatability of point source measurements is severely degraded Thus moderate or good "seeing" is a necessity even at $10 \mu \mathrm{m}$.

\section{RECENT ASTRONOMICAL RESULTS}

Tables 2 and 3 provide a summary of our observations of several classes of stars at various wavelengths Table 3 lists stars where only upper limits have been obtained Also listed are stars frequently used as point sources The column labeled $\theta_{M}$ gives the "minimum angular diameter" calculated as follows The spectral energy distribution of the infrared excess is fitted to a Planck distribution to give an approximate color temperature, Tc; the angular diameter of a uniform disk at that temperature is calculated to give the observed flux at $10 \mu \mathrm{m}$. This serves only as a guide for what we may expect of a given star since it neglects a number of factors such as the optical properties of the dust and the radial distribution and shape of the circumstellar envelope. It points out that for bright objects with typical color temperatures in the range of 300-600K the size of interest is $0.2-2.0$ arcsec

Not only have IRC +10216 , VY CMa and NML Cyg been resolved but their shapes have been measured as well These results are discussed in the paper by $D$. McCarthy at these proceedings

Betelguese, the brightest $3000 \mathrm{~K}$ supergiant in the sky, shows an infrared excess beyond $10 \mu \mathrm{m}$. Our objective has been to resolve the circumstellar dust shell or cloud at as many wavelengths as possible in order to study what was initially thought to be a simple case of "silicate" dust emission. Figure 3 summarizes our date from $1977,{ }^{3}$ the published Berkeley data, 4 and new observations at $12.8 \mu \mathrm{m}$. At wavelengths short of $10 \mu \mathrm{m}$ the star is unresolved but at 10.2, 11 , and $12.5 \mu \mathrm{m}$ the dust envelope is completely resolved and it is now possible to unambiguously separate the spectral energy distribution of the dust from that of the star itself. It is interesting that the unresolved flux from the star is greater than that from a $3000 \mathrm{~K}$ photosphere.

Mira is another star which has been extensively observed by both the Arizona and Berkeley groups. Figure 4 shows our recently published results 5 


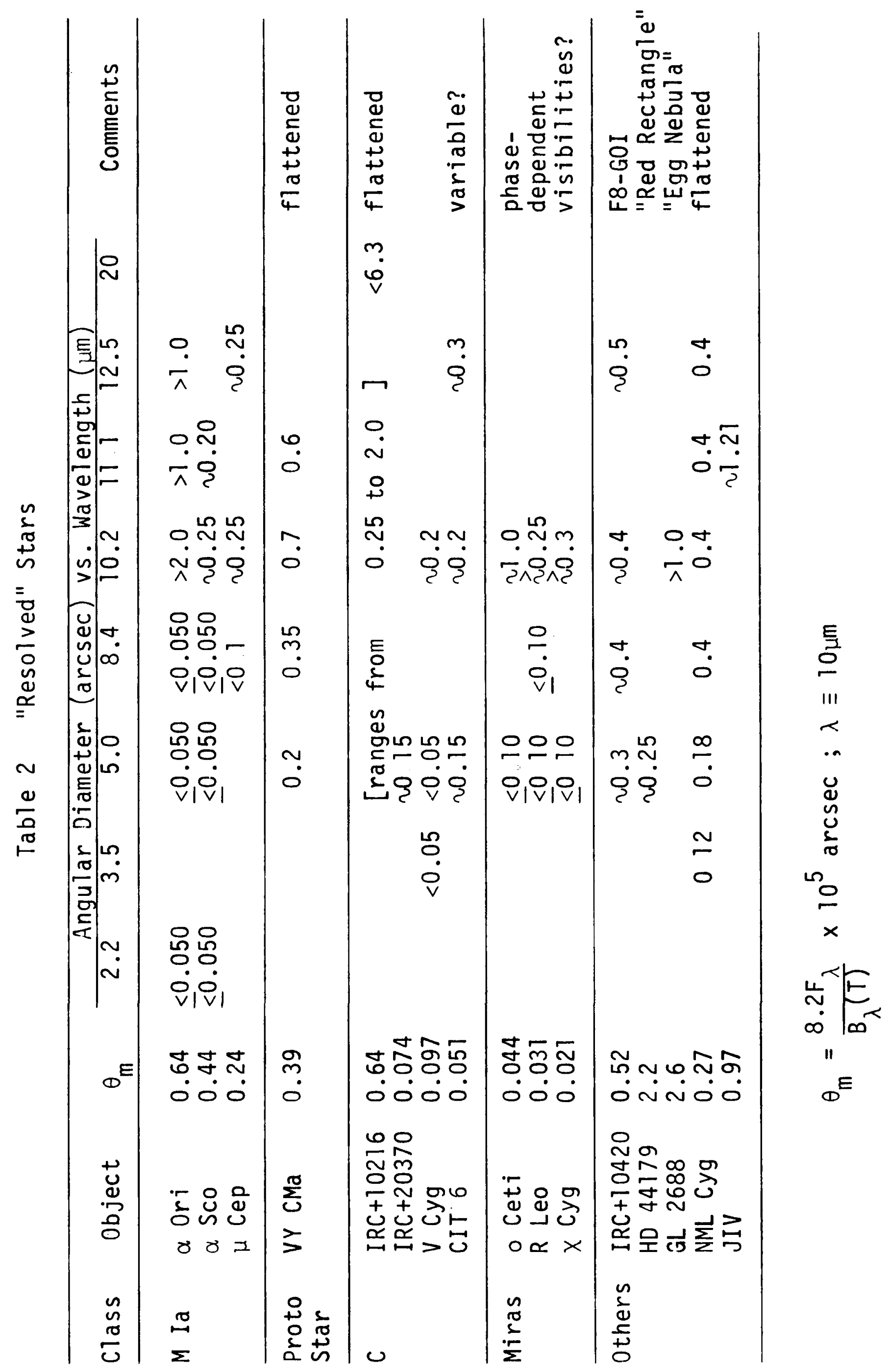


Table 3. Other Objects

\begin{tabular}{lll}
\hline \multicolumn{2}{c}{ Unresolved Stars } & Point Sources \\
\hline T Cas & Lk Ha-101 & $\alpha$ Lya \\
IRC +10011 & TX Cam & $\alpha$ Her \\
S Per & R Lep & $\alpha$ Tau \\
Beck1in-Neugebauer & V Hyd & $\alpha$ Peg \\
W Hyd & R Hyd & $\alpha$ Boo \\
RX Boo & AH Sco & $\alpha$ CMa \\
VX Sgr & MWC 297 & $\alpha$ SCO \\
MWC 349 & GL 3068 & \\
\hline
\end{tabular}

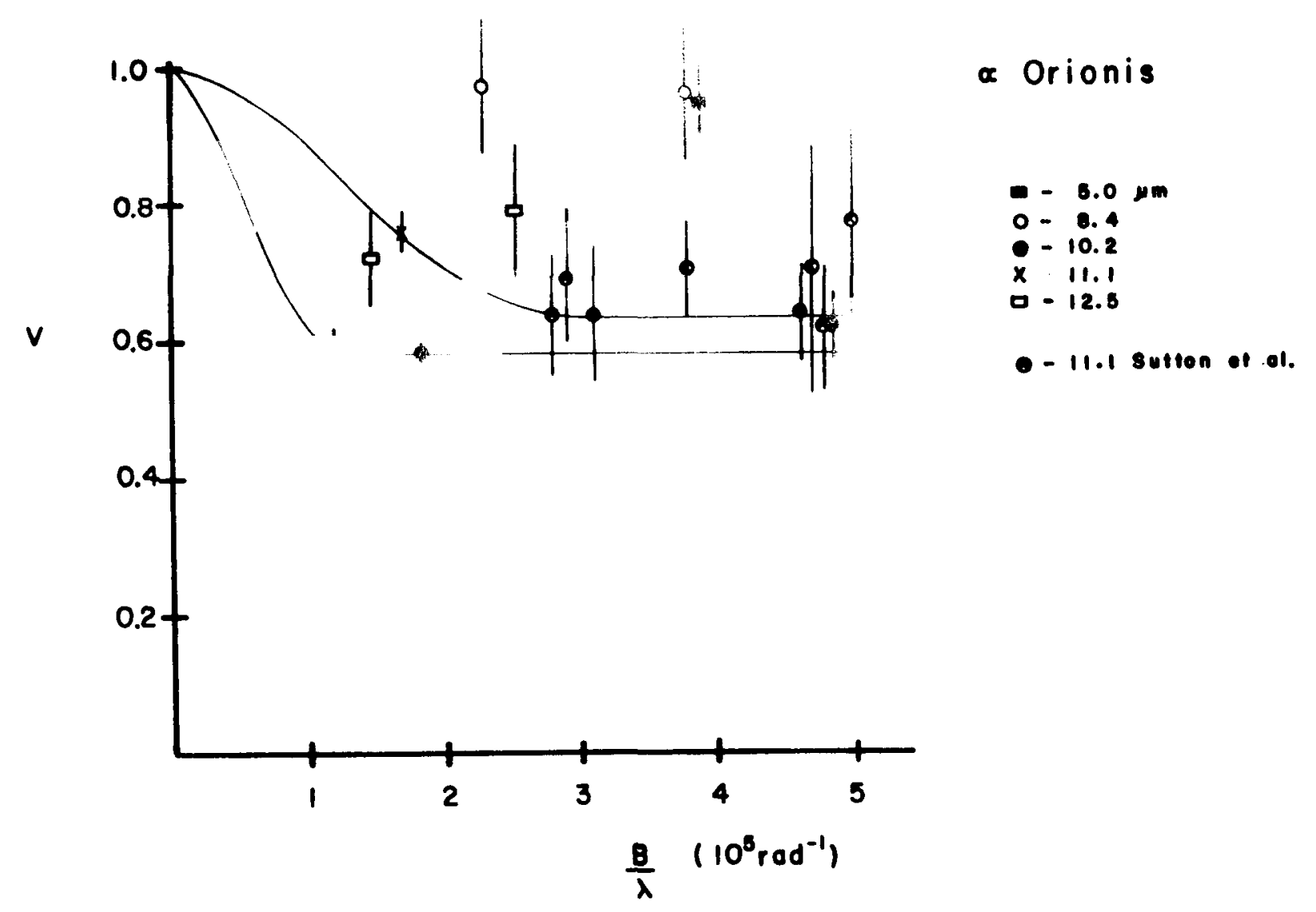

Figure 3 Visibility of $\alpha$ Ori at several wavelengths showing the large resolved dust envelope emitting $\sim 40 \%$ of the continuum beyond $10 \mu \mathrm{m}$ 


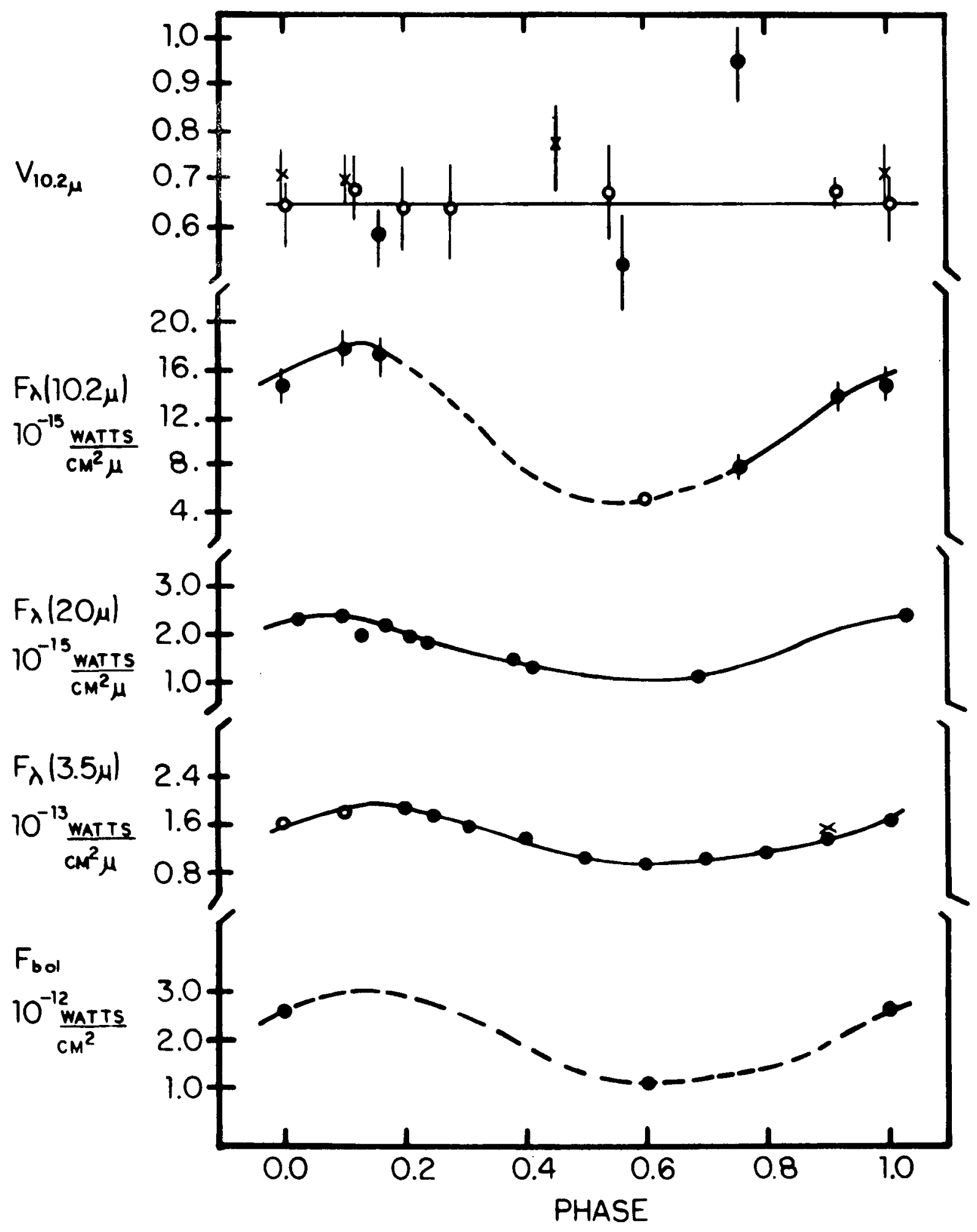

Figure $4 \quad V_{10.2 \mu}$ is plotted for various phases of Mira Recent unpublished data appear to show a nearly constant visibility at the observed baselines of $1.2,19$ and 3.2 meters 
and new measurements obtained during the period when Mira was a daytime object. It now seems clear that our high visibility measurements at phase 0.75 must be in error and that the visibility at baselines less than $3.2 \mathrm{~m}$ is nearly independent of phase.

In a variable star such as Mira, where the radiant input into the circumstellar dust varies a factor of about 2.5 as shown in Figure 4 , one would expect to see changes in the visibility function caused by two effects (1) heating and cooling of the dust and (2) changes in contrast between the unresolved core and the resolved envelope. The first effect should be detected at baselines between 1 and 2 meters where the dust is only partially resolved and should be greatest at phase 11 where the $10 \mu \mathrm{m}$ emission reaches its maximum. Our data indicate that this effect is small and will require higher accuracy to be detected. The second effect, a change in contrast between the resolved and unrevolved components should be best detected at baselines beyond 2 meters where the dust envelope is completely resolved

Table 2 gives results for two other bright Mira stars, X Cyg and R Leo. Both are resolved and show possible signs of variable visibilities Observations of these three stars are being continued

\section{INSTRUMENTAL IMPROVEMENTS}

There are at least three well understood deficiencies in our apparatus at $\lambda>8 \mu \mathrm{m}$ : (1) the bolometer is too small to detect all the energy diffracted by the grid (2) because of the high frequency response required and the relatively narrow bandwidth used, the bolometer is not background limited (3) the geometry inside the dewar does not allow the detector to be optimally shielded from background

By using a newly developed diamond-germanium composite bolometer cooled to $0.3 \mathrm{~K}$ by a $\mathrm{He}^{3}$ cryostat and a much larger dewar it should be possible to solve all three of these problems and the sensitivity should approach the limiting value given in Table 1 
Two avenues for improving the accuracy and reliability of the measurements should be explored In principle it is possible to measure both the photometric and interferometric signals simultaneously, thus reducing the effects of guiding and setting errors and saving observing time We have already demonstrated the benefits of offset guiding on the 4-meter telescope At least some of the effects of poor "seeing" might be reduced if motion of the two images were sensed at shorter wavelengths and reduced by servo-control on a rapid time scale. Preliminary studies indicate that this method might be useful to improve accuracy on bright stars

\section{THE MMT}

Figure 5 shows the optical diagram of the MMT, which, when completed late in 1978 or early in 1979, will be the largest optical/infrared telescope in the world measured across its aperture The six 1.8 meter primary mirrors provide a collecting area equivalent to a 4.4 meter single mirror telescope but can be configured to simultaneously provide three two-element interferometers of almost 7 meters separation Table 4 summarizes the resolution as a function of wavelength for the telescopes now used and for the MMT.

Table 4 Angular Resolution vs Baseline and Wavelength

\begin{tabular}{|c|c|c|c|c|c|}
\hline Telescope (m) & Baseline $(\mathrm{m})$ & $\lambda=2.0$ & $\begin{array}{c}\text { Gaussian } e^{-1} \\
\text { Diameter at } V=0 \\
5.0\end{array}$ & $\begin{array}{l}\text { Angula } \\
90 \text { in } \\
10.0\end{array}$ & $\begin{array}{l}\operatorname{ar} \\
\operatorname{arcsec} \\
20.0 \mu \mathrm{m}\end{array}$ \\
\hline 1.02 & 0.9 & 0.094 & 0.24 & 0.47 & 0.95 \\
\hline 155 & 1.2 & 0.071 & 018 & 0.35 & 0.71 \\
\hline 2.29 & 1.9 & 0.045 & 011 & 0.22 & 0.45 \\
\hline 3.81 & 3.2 & 0.027 & 0.067 & 0.13 & 0.27 \\
\hline 6.9-MMT & 6.5 & 0.013 & 0.032 & 0.065 & 0.13 \\
\hline
\end{tabular}



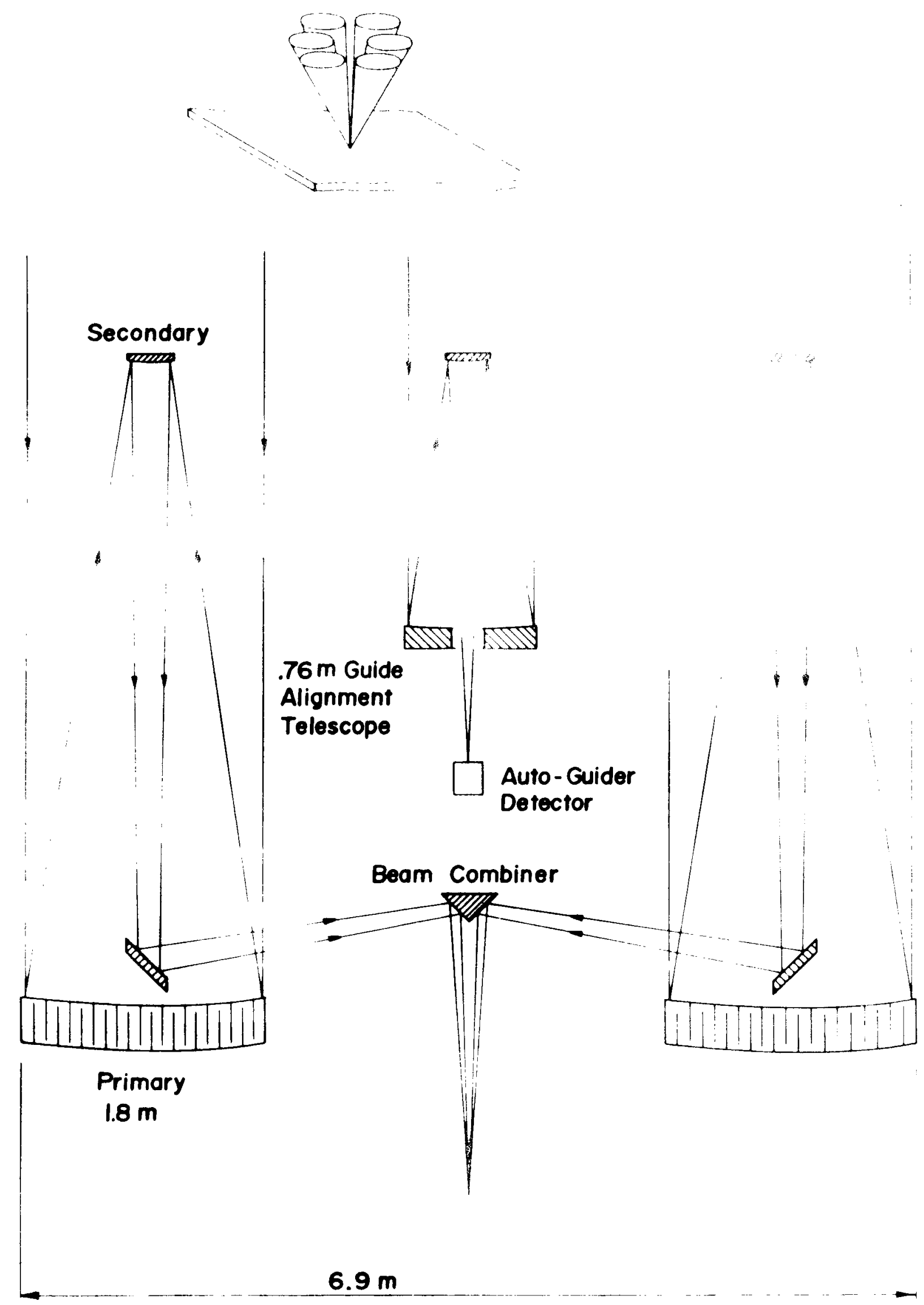

Figure 5: Optical diagram of the 6.9 meter Multiple Mirror Telescope 


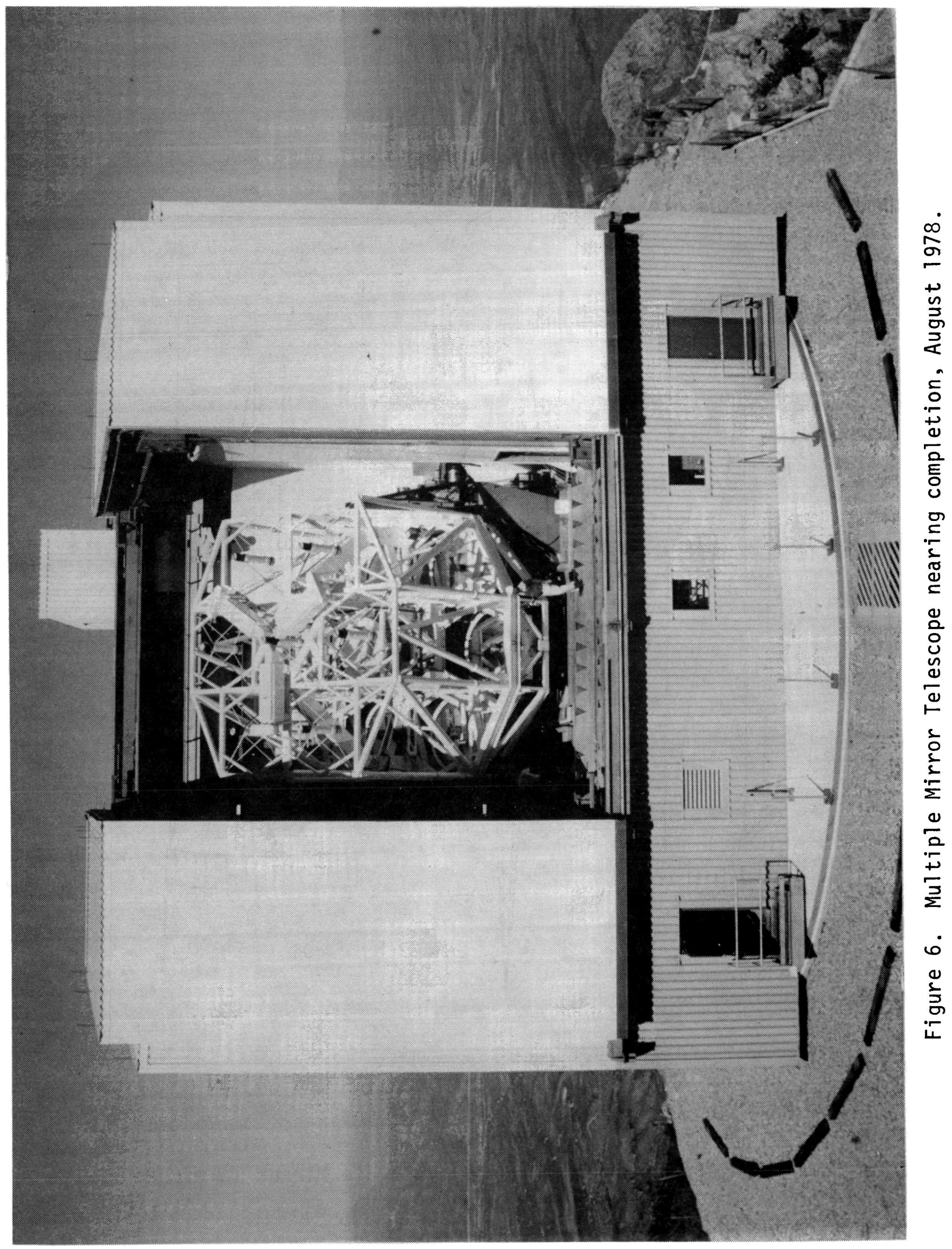


Figure 6 shows the facility as it neared completion in mid 1978. With its laser stabilized active optics control system, this telescope should become an important tool for high angular resolution studies at infrared wavelengths.

\section{ACKNOWLEDGEMENTS}

This research is supported by the National Science Foundation

\section{REFERENCES}

1) McCarthy, D.W, and Low, F.J Ap.J (Letters), 202, L37, 1975

2) McCarthy, D.W, Low, F.J , and Howell, R. Opt Eng , 16, 569, 1977

3) McCarthy, D.W., Low, F.J., and Howe11, R. Ap.J (Letters), 214, L85,

4) Sutton, E.C , Storey, J.W.V , Betz, A.L, Townes, C.H , and Spears, D.L. Ap.J. (Letters), 217, L97, 1977

5) McCarthy, D.W, Howel1, R, and Low, F.J , Ap.J (Letters), 223, L113, 1978

\section{DISCUSSION}

D.D. Cudaback: Have you explored how the maximum usable aperture goes with wavelength?

F. J. Low: With regard to coherence over the aperture as a function of wavelength, we have data but I wouldn't like to be too specific at this stage. Our largest aperture has been $0.6-0.7 \mathrm{~m}$ and when the visual seeing is $<3$ arc-seconds the fringes track nicely and the amplitude of the fringes behaves well enough to give results in the region of $\sim 10 \%$. For visual seeing worse than 3 arc-seconds things change drastically and the effects of rapid changes in phase, of differential tilts resulting in lack of image superposition, and perhaps loss of coherence over the apertures, all combine at about the same point to degrade the measurements. 